\title{
Type IV collagen and laminin in Bruch's membrane and basal linear deposit in the human macula
}

\author{
G E Marshall, A G P Konstas, G G Reid, J G Edwards, W R Lee
}

\begin{abstract}
Tissue obtained from the macula in $\mathbf{1 0}$ human eyes (53-77 years) was used for an investigation into the extracellular matrices of the retinal pigment epithelium (RPE), Bruch's membrane, and the choriocapillaris. The ultrastructural distribution of type IV collagen and laminin was documented using immunogold labelling. Labelling for type IV collagen was strongly positive in all the specimens in the basement membranes of the choriocapillaris but not that of the RPE where labelling was either weak or absent. Laminin was localised to deposits of granular material in Bruch's membrane but was absent from the basement membrane of the RPE and the choriocapillaris. Basal linear deposit, observed in three cases, demonstrated labelling for laminin but not for type IV collagen. The series was too small for correlation of these morphological changes with age.
\end{abstract}

(Brf Ophthalmol 1992; 76: 607-614)

Vision can be affected in the elderly by a variety of pathological changes which interfere with the function of the retinal pigment epithelium (RPE) and the maintenance of the photoreceptors. Photoreceptor atrophy occurs when an abnormal extracellular matrix - for example, drusen, accumulates between the pigment epithelium and Bruch's membrane. Drusen are clinically well recognised and can be classified as hard, soft, calcified, and diffuse according to histological criteria. ${ }^{1}$ However, a morphological distinction can be made between drusen and basal linear deposit. Various authors ${ }^{2-4}$ have used the term 'basal linear deposit' for an accumulation with specific appearances (after PAS and Mallory trichrome stains) beneath the RPE. The precise biochemical composition of the basal linear deposit is not known and identification of the constituents is essential to an understanding of the pathogenesis of age-related macular degeneration.

Studies using transmission electron and light microscopy have adequately documented the morphology of the age-related changes in Bruch's membrane. ${ }^{25-8}$ The initial change is an accumulation of vesicles and granular and filamentous material in the inner collagenous zone. This accumulation progressively increases with age until it occupies both collagenous zones. Various attempts have been made to determine the biochemical nature of the age-related deposits in Bruch's membrane outside the macular region $^{9-13}$ but it is not known if their accumulation is the cause or the result of RPE dysfunction. Since Bruch's membrane may act as a selective barrier to the movement of molecules from the choroidal circulation to the outer retina, ${ }^{14}$ alterations in its composition could have important physiological consequences.

Age-related thickening and degeneration in Bruch's membrane, ${ }^{715}$ drusen formation, ${ }^{16}$ and basal linear deposits ${ }^{3}$ each appear to provide a matrix which stimulates ingrowth of macrophages and endothelial cells. Vascular ingrowth is an important complication of age-related macular degeneration and is a major cause of blindness in the elderly population of the Western world. ${ }^{118}$ Bruch's membrane acts as a barrier to subretinal neovascularisation and breaks in this structure significantly increase the incidence of growth of new vessels. ${ }^{7}$ The differences between the factors causing senile degenerative atrophy and disciform degeneration are poorly understood. ${ }^{4}$ It is now accepted that type IV collagen and laminin are matrix constituents which facilitate endothelial cell movement in vitro. ${ }^{19-22}$ Thus knowledge of the precise biochemical composition of the age-related deposits in Bruch's membrane will allow an evaluation of their potential influence on vascular ingrowth in disciform degeneration. As early forms of basal linear deposit have ultrastructural similarities to basement membrane ${ }^{2}$ it was considered likely that the deposit would contain type IV collagen and laminin which are the principal components of many basement membranes. In this study we investigated the fine structural distribution of collagen type IV and laminin in Bruch's membrane of aged human macula.

\section{Materials and methods}

Normal aged macular tissue was obtained both from freshly enucleated eyes of five uveal melanoma patients, a patient with a corneal ulcer, and from four postmortem eyes. The age range of the subjects was between 53 and 77 years (Table 1). Routine macroscopic and microscopic examination excluded the presence of any secondary disease process which could have affected macular morphology.

Surgically enucleated eyes were fixed

Table 1 Clinical details of surgical enucleations and postmortem eyes

\begin{tabular}{|c|c|c|c|c|}
\hline Case no & Age & Sex & Indicat & on for enucleation \\
\hline $\begin{array}{r}1 \\
2 \\
3 \\
4 \\
5 \\
6 \\
7 \\
8 \\
9 \\
10\end{array}$ & $\begin{array}{l}53 \\
54 \\
58 \\
59 \\
60 \\
64 \\
71 \\
73 \\
75 \\
77\end{array}$ & $\begin{array}{l}F \\
F \\
M \\
M \\
M \\
M \\
F \\
M \\
F \\
F\end{array}$ & $\begin{array}{l}\text { CBM } \\
\text { CBM } \\
\text { PM } \\
\text { PM } \\
\text { PM } \\
\text { PM } \\
\text { PM } \\
\text { CM } \\
\text { CBM } \\
\text { CM }\end{array}$ & $\begin{array}{l}\text { BLD } \\
\text { BLD } \\
\text { BLD }\end{array}$ \\
\hline
\end{tabular}

$\mathrm{CBM}=$ ciliary body melanoma; $\mathrm{CM}=$ choroidal melanoma; $\mathbf{P M}=$ postmortem; $\mathbf{B L D}=$ basal linear deposit.

\author{
Dr G E Marshall, Department \\ of Ophthalmology, University \\ 6NT. \\ Accepted for publication \\ 23 April 1992 \\ Ophthalmology, Cell \\ University of Glasgow, \\ G E Marshall \\ G G Reid \\ J G Edwards \\ W R Lee
}


immediately after enucleation in freshly prepared fixative of $4 \%$ paraformaldehyde (PFA) plus $0 \cdot 2-1 \cdot 0 \%$ glutaraldehyde in $0 \cdot 1 \mathrm{M}$ Sorensen's phosphate buffer or cacodylate buffer ( $\mathrm{pH} \mathrm{7 \cdot 4)}$ with $5 \%$ sucrose added; details of fixation are provided elsewhere. ${ }^{23}$ The postmortem eyes were obtained from the Greek Eye Bank (University Department of Ophthalmology,

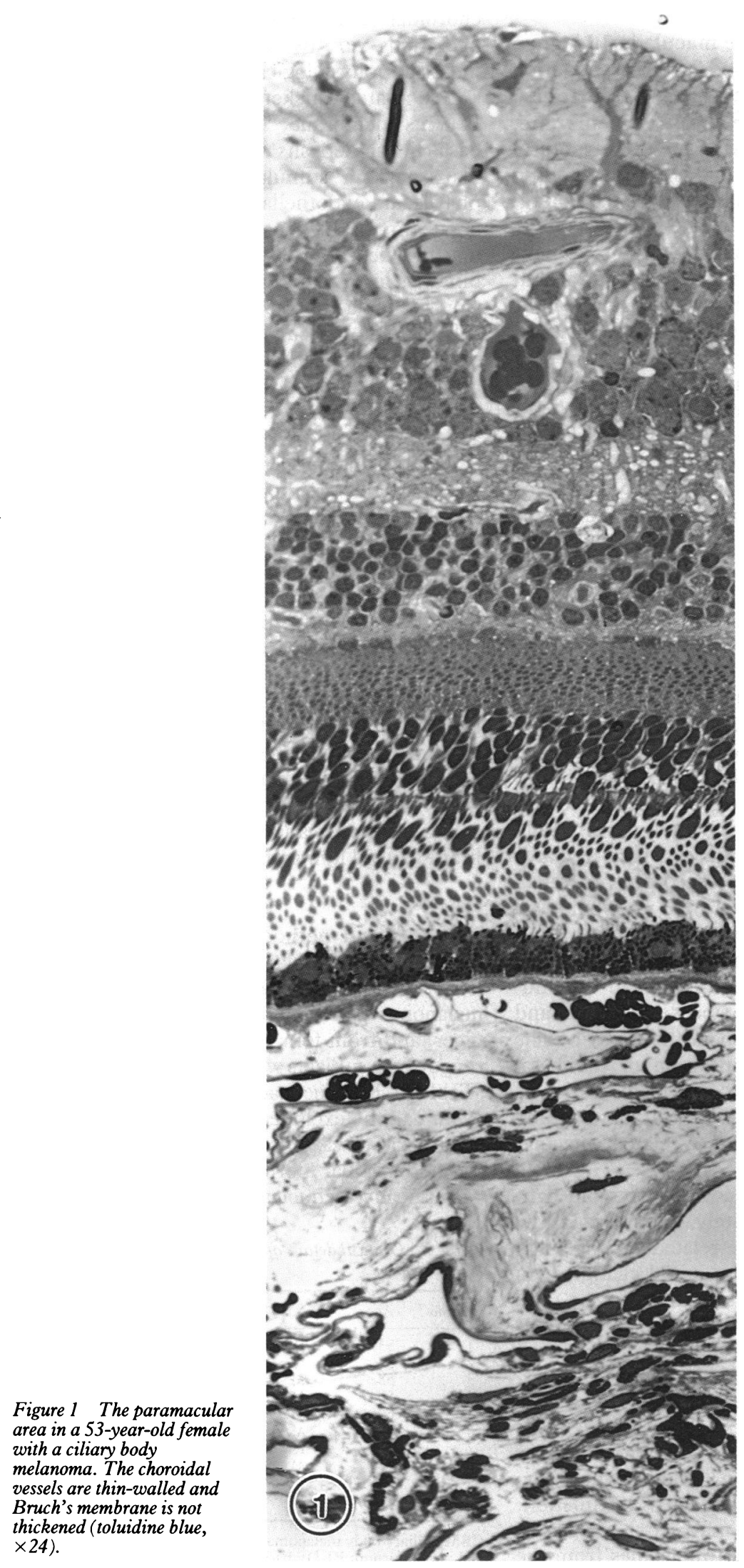

Thessaloniki) and fixed within 1.5 to 9 hours of death following removal of the cornea for transplantation. Fixation was achieved with $0.5 \%$ glutaraldehyde plus $4 \%$ formalin in phosphate buffer $\mathrm{pH} \mathrm{7.4} \mathrm{at} \mathrm{room} \mathrm{temperature}$ for 2 hours. Details of the medical and ocular history of the postmortem cases were obtained from close relatives and subsequent macroscopic examination revealed a normal macula and disc.

The macula region was dissected from each eye and in the majority of cases the retina remained attached. Radial blocks were taken and processed for London resin white (LR white) embedding and cryoultramicrotomy. ${ }^{23} \mathrm{LR}$ white is a hydrophilic resin that is used for immunocytochemical studies owing to its property of preserving tissue antigenicity. ${ }^{24}$

IMMUNOCYTOCHEMISTRY

Polyvalent goat antibodies against type IV collagen were raised against human and bovine type IV collagen and supplied by Southern Biotechnologies (Bionuclear Services Ltd). The antibody was affinity purified and cross absorbed against types I, II, III, and V collagen which were purified from human tissue. The specificity of this antibody to human type IV collagen has been confirmed by the supplier using indirect enzyme linked immunosorbent assay (ELISA).

Polyvalent rabbit antibodies against laminin were supplied by Heyl (Germany) and had been raised in rabbits by multiple injections of human laminin. The serum has been tested by the supplier with the following immunological assays: ELISA, immunoblot, and immunohistology (fluorescence, APAAP). The antibody dilutions in Tris buffer plus $1 \%$ BSA, determined by previous experimental work, were between $1: 40$ and $1: 150$ for type IV collagen and laminin antibodies at dilutions between $1: 30$ and $1: 100$. These antibodies have been successfully employed in our laboratory for immunogold electron microscopic studies of various ocular tissues. ${ }^{25-28}$ The precise immunocytochemical procedure has been described by Marshall et al. ${ }^{27}$

\section{CONTROLS}

Negative controls comprised substitution of the primary antiserum with normal goat and rabbit serum (Sigma) at the same dilutions. Retinal vessels were used as an internal positive control (within the same tissue section) for type IV collagen which is located in the basement membranes within the vessel wall. ${ }^{25}$ The basement membranes of the ciliary epithelium were used as an external positive control for type IV collagen and laminin. ${ }^{28} 29$ We consider internal positive controls to be even more reliable than external positive controls from another tissue block.

Our criteria for positive localisation required the restriction of immunogold particles to discrete structures. Labelling was considered to be non-specific if a significant number of immunogold particles were present on internal negative controls. Internal negative controls comprised cell nuclei, mitochondria, pigment granules, and red blood cells: on the basis of 


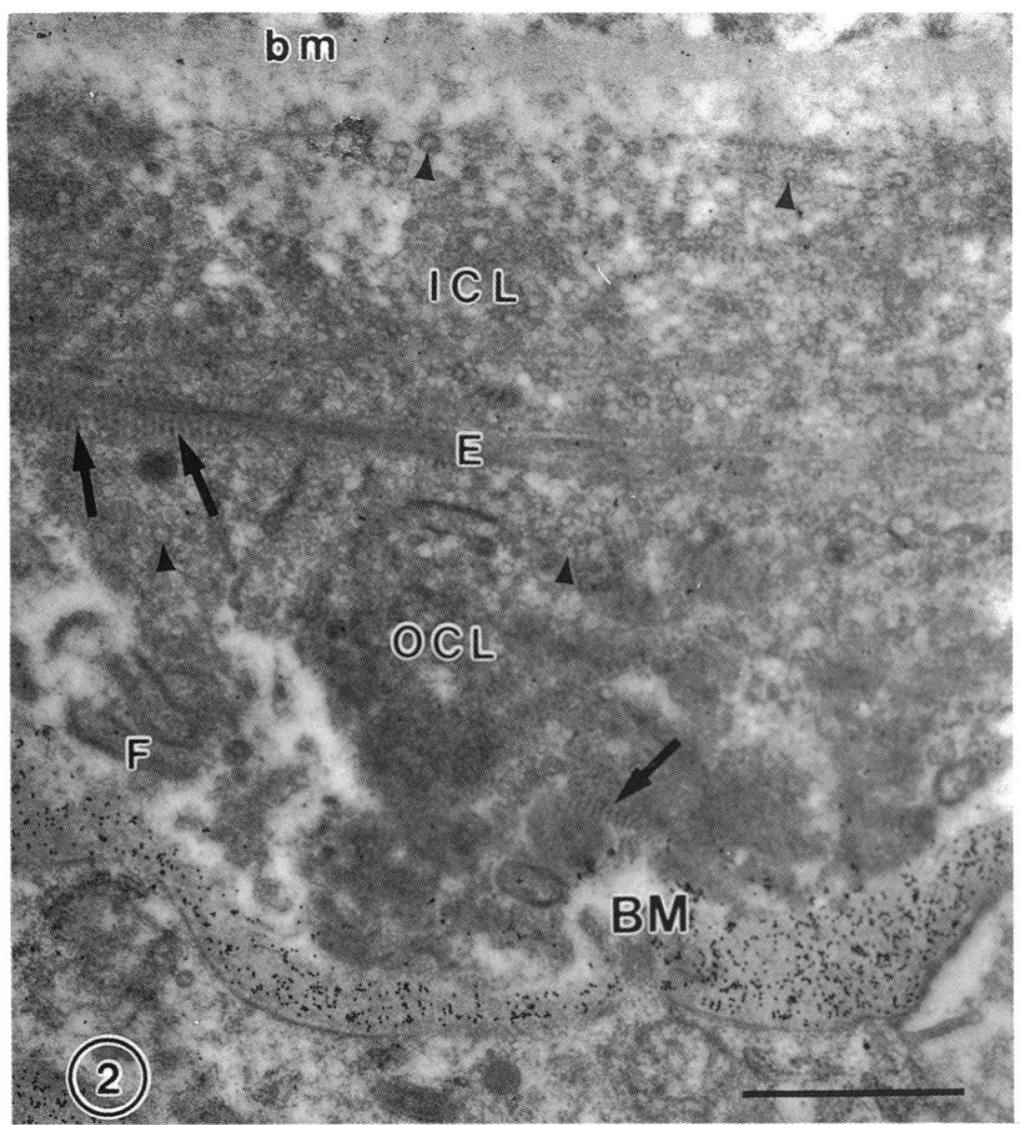

Figure 2 Type IV collagen distribution in Bruch's membrane. Immunogold particle density over the RPE basement membrane $(b m)$ is considerably less than that over the basement membrane (BM) of the choriocapillaris. A small amount of type IV collagen is also present in the inner collagenous layer (ICL), the elastic layer $(E)$, and the outer collagenous layer $(O C L)$, that of the elastic layer occurring in a space between elastic fibrils. Note association of fibrous

banded material with elastic layer as well as its presence in the outer collagenous layer (arrows). Coated vesicle-like bodies can be seen in both the inner and outer collagenous layers

(arrowheads). Coated membrane fragments $(F)$ are restricted to the outer collagenous layer $($ bar $=1 \mu \mathrm{m})$.

Figure 3 Fine structural localisation of laminin in Bruch's membrane.

Immunogold particles are absent from the basement membranes of the RPE (bm) and the choriocapillaris $(B M)$. Some degree of labelling is present in the inner collagenous layer (ICL) particularly at its interface with the RPE basement membrane

(arrowheads). Note the absence of immunogold particles from the elastic layer $(E)$. Labelling of the outer collagenous layer $(O C L)$ is concentrated mainly to granular deposits in the region of its interface with the choriocapillary

basement membrane (BM).

Coated vesicle-like bodies

$(V)$ and coated membrane

bound bodies $(M)$ are free of label (bar=1 $\mu \mathrm{m}$.) Inset $A$ : Laminin labelling at the interface between the RPE basement membrane (bm) and the inner collagenous layer was of ten restricted to small electron dense plaques (bar $=1 \mu \mathrm{m}$ ). Inset $B$ : Immunogold particles localising laminin are largely restricted to the periphery of fibrous banded materia (bar $=0.5 \mu \mathrm{m}$ ) previous investigations these had been found to be the first structures to exhibit non-specific labelling.

\section{Results}

The use of different concentrations of glutaraldehyde in surgically enucleated eyes did not alter the pattern or intensity of immunogold labelling for type IV collagen and laminin. The quality of preservation of cells in postmortem specimens was poorer than that in surgically enucleated eyes, but in all the specimens the structure was morphologically satisfactory by light microscopy (Fig 1). The addition of 5\% sucrose to the cacodylate buffer eliminated the previously documented superiority of phosphate buffer in ultrastructural preservation. ${ }^{26}$

There was no apparent difference in immunolabelling of collagen type IV and laminin when comparing sections from LR white processed tissue with ultrathin sections of frozen tissue. LR white embedding was predominantly used because it was much less technically demanding than cryoultramicrotomy and more satisfactory in terms of preservation of ultrastructure in the material used in the present study. Our previous studies in aged ocular tissues have demonstrated similar results with both techniques for laminin and type IV collagen. Therefore for the study of laminin and type IV collagen, LR white embedding was considered the technique of choice. The employment of both techniques served to consolidate the results. However, the illustrations for the present communication were prepared from LR white embedded material.

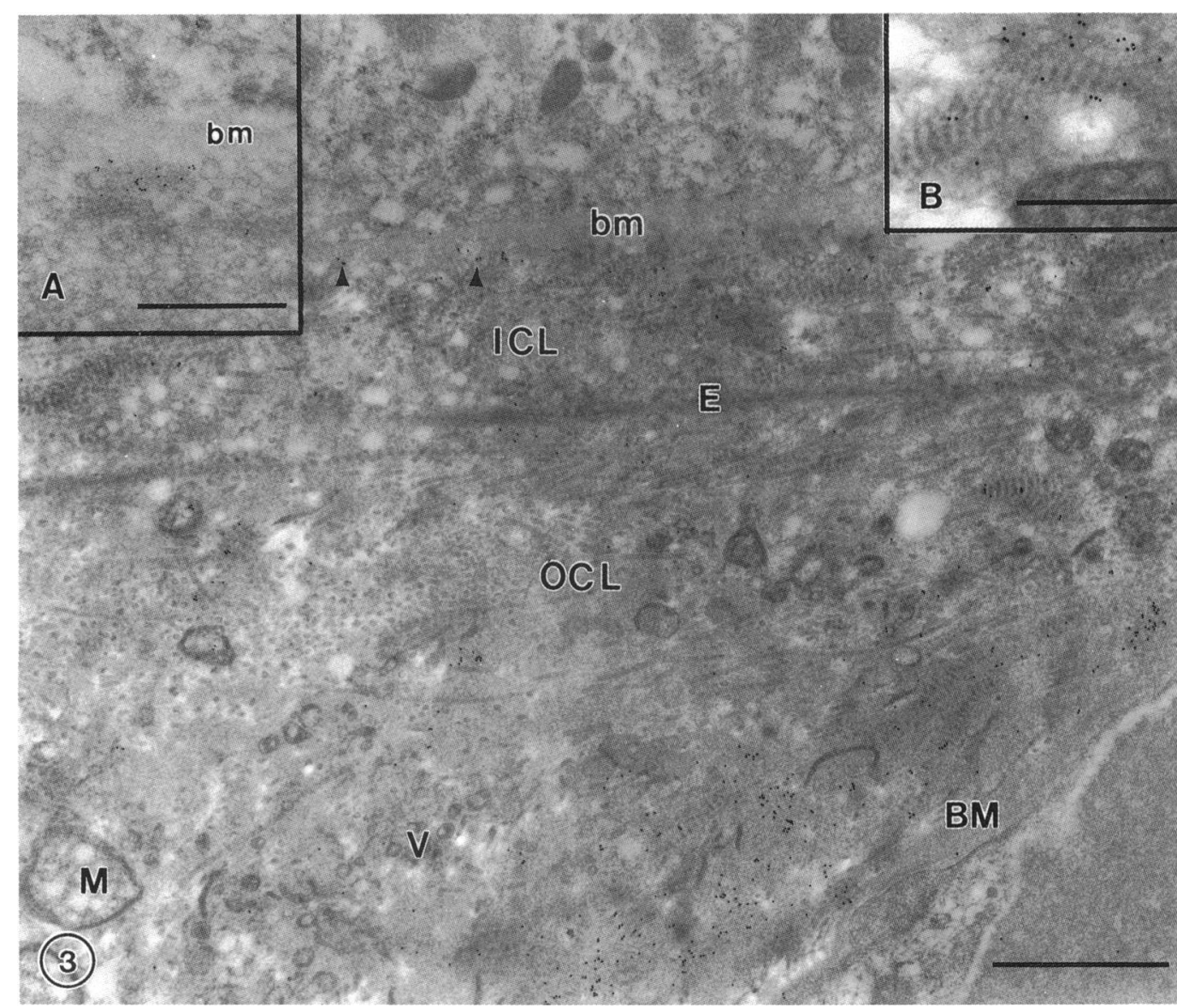




\section{NOMENCLATURE}

In the description of type IV collagen and laminin distribution in Bruch's membrane we have adopted the nomenclature proposed by Killingsworth ${ }^{5}$ to classify the various deposits associated with aging of Bruch's membrane. According to this classification 'coated vesiclelike bodies' are circular bodies composed of a single membrane lining an electronlucent core and occurring in two sizes $(70 \mathrm{~nm}$ and $110 \mathrm{~nm})$. 'Coated membrane-bound bodies' (400 to 2500 $\mathrm{nm}$ diameter) contain fine granular material, droplets, and coated vesicle-like bodies. 'Coated membrane fragments' are thought to be the remnants of ruptured coated membrane-bound bodies. $^{5}$

\section{IMMUNOCYTOCHEMISTRY}

\section{Basement membrane of the RPE}

In some specimens the basement membrane of the RPE exhibited weak labelling with type IV collagen antibodies (Fig 2) but this finding was not uniform in all the cases studied as labelling was absent from other specimens. Such variation in labelling could not be related to the source of tissue (that is, surgical enucleations or postmortem specimens) nor to the concentration of fixative employed. The most surprising finding was that laminin was not identified in the RPE basement membrane in any of the specimens investigated (Figs 3-6).

\section{Bruch's membrane}

A small amount of labelling for type IV collagen was present in the inner collagenous layer, the elastic layer, and the outer collagenous layer of Bruch's membrane. This was a consistent feature in all the specimens studied. Although labelling was present in the elastic layer, immunogold particles were not located to the elastic fibres but rather to the matrix surrounding these fibres (Fig 2). Immunogold labelling for type IV collagen was absent from coated vesicle-like bodies, coated membrane bound bodies, coated membrane fragments, and fibrous banded material. Laminin was localised to deposits of fine granular-like material present in the inner collagenous layer. More frequently laminin was dispersed within the fine granular-like material between the outer collagenous layer and the basement membrane of the choriocapillaris (Fig 3). Small discrete patches of laminin labelling were also observed in the inner collagenous layer immediately adjacent to the RPE basement membrane (Fig 3, inset A). These patches of labelling were spaced in a manner which was almost regular. The only difference in the structure of these patches and the inner collagenous layer was a small augmentation in electron density.

Laminin was also associated with the periphery of fibrous banded material present within Bruch's membrane, labelling being generally absent from the actual striations of this material (Fig 3, inset B). We considered that fibrous banded material was quite distinct from long-spacing collagen in its location, structure,

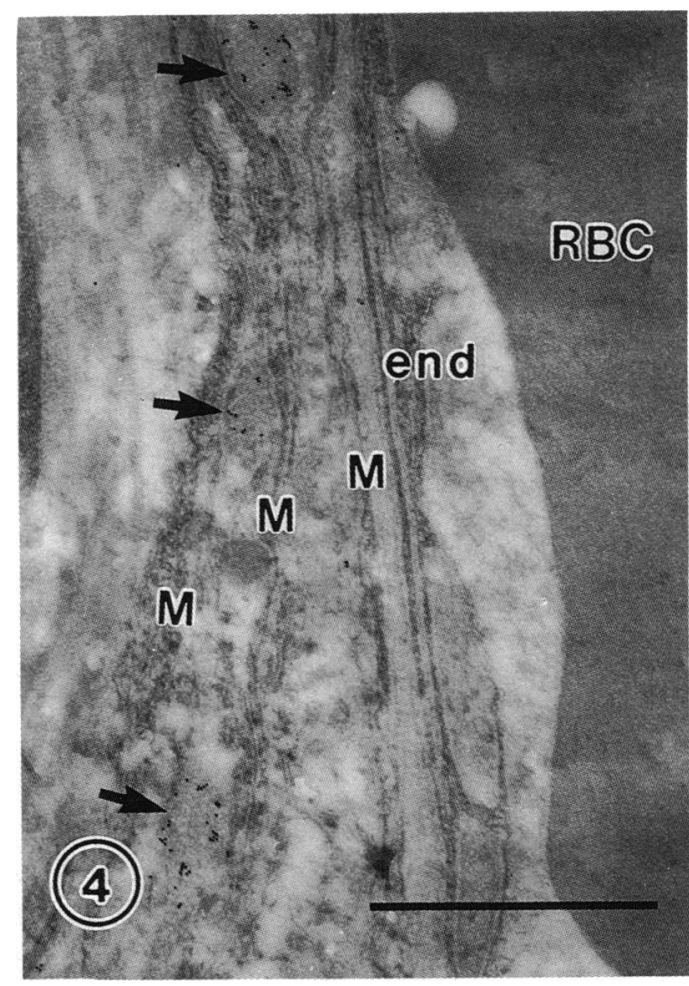

Figure 4 Laminin distribution in major blood vessel in the choroid. Laminin localised to discrete clumps of granular-like material (arrowed) between the three layers of myocytes $(M)$ within the vessel wall. Note absence of label from red blood cell $(R B C)$. (end=endothelium of blood vessel $($ bar $=1 \mu \mathrm{m})$.

and periodicity. Long-spacing collagen was present between the outer collagenous layer and the basement membrane of the choriocapillaris. Fibrous banded material was located within both the inner and outer collagenous layers and sometimes in association with the elastic layer (Fig 2).

In contrast with long-spacing collagen, fibrous banded material had a significantly shorter periodicity and lacked the fine structure of the latter. No association of laminin was seen with long spacing collagen within Bruch's membrane. Similarly, laminin was absent from the elastic fibres in the elastic layer (Fig 3).

\section{Choriocapillaris}

There was considerable degree of variation in the thickness of the basement membrane of the endothelial cells and this could not always be attributed to the plane of section of the choriocapillaris. Type IV collagen was clearly localised to the basement membranes around the circumference of the choriocapillaris and labelling was present throughout the entire width of the basement membrane. In contrast to the RPE basement membrane, the basement membrane of the choriocapillaris exhibited intense labelling with type IV collagen antibodies (Fig 2). Antibody labelling for laminin was so sparse as to be regarded as negative.

\section{Choroidal stroma}

Type IV collagen and laminin were localised to the basement membranes of the large choroidal vessels. Type IV collagen was also localised to all basement membranes present in the stroma, some of which were not associated with cells but 
Figure 5 Immunogold localisation of laminin in early form of basal linear deposit. Labelling is restricted to the circumference of this amorphous basement membrane-like material which has not yet separated the retinal pigment epithelium ( $R P E)$ from its basement membrane $($ bar $=1 \mu \mathrm{m})$.

Figure 6 Laminin present within flamentous form of immature basal linear deposit (arrowed) (bar $=1 \mu \mathrm{m}$ ).

Figure 7 Early stage basal linear deposit exhibiting granular-like morphology is labelled throughout with antilaminin antibodies. Note numerous basal infoldings separating deposit from basement membrane (bm) of retinal pigment epithelium (RPE). Note absence of laminin from RPE basement membrane $($ bar $=1 \mu \mathrm{m})$.
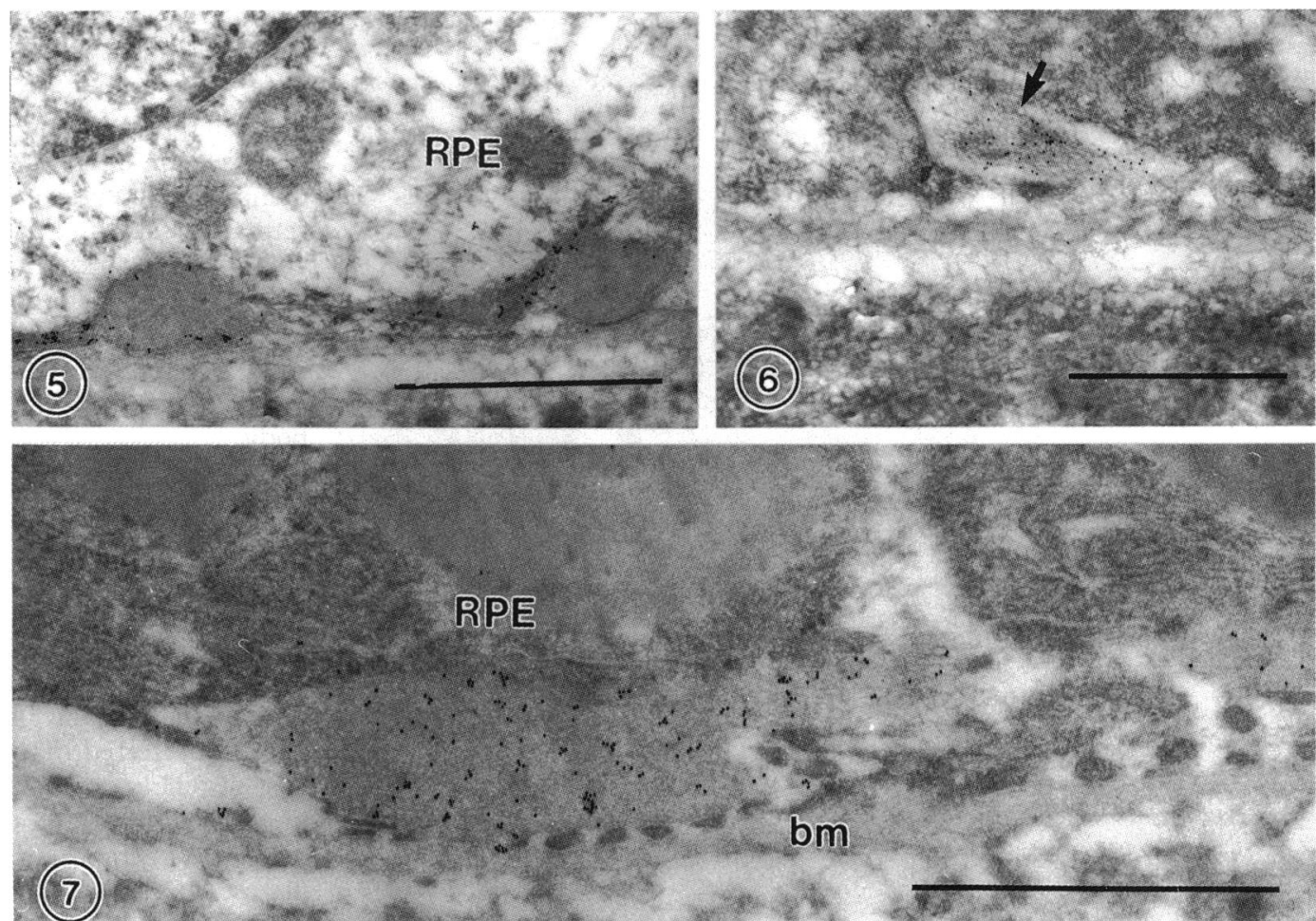

were lying free amongst collagen fibrils of the stroma. Small isolated deposits of fine granular material, similar in appearance to basal linear deposit (see below), were occasionally seen in the stroma between large blood vessels and were positive for laminin (Fig 4). Elastic fibres within the walls of large blood vessels were free of immunogold particles for laminin or type IV collagen. Considerably larger deposits of granular material similar to those observed in Bruch's membrane were frequently present in the intercapillary zone. These deposits appeared to be associated with choroidal stroma rather than the outer collagenous layer.

\section{Basal linear deposit}

Basal linear deposit was observed in three out of the 10 specimens (cases $6-8$, see Table 1 ). Small sized basal linear deposit could be divided into three types on the basis of their morphological appearance: basement membrane-like, granular- like, and filamentous-like. These deposits were within basal infolds and were not accompanied by a separation of the RPE from its basement membrane. Two distinct labelling patterns of these deposits were then noted with laminin antibodies. Labelling for laminin was restricted to the margins of the basement membrane-like basal linear deposit (Fig 5) but was present throughout the entire matrix of the granular-like (Fig 6) and fine fibrillar-like structures (Fig 7).

Larger forms of basal linear deposit contained a mixture of basement membrane-like, fine granular-like, and fine fibrillar-like material. These deposits were invariably accompanied by a separation of the RPE from its basement membrane (Fig 8). The labelling pattern of the large deposits for laminin was similar to that of the small deposits: the margins of basement-like material and the entire matrix of the fine granular-like and fine fibrillar-like material were labelled (Fig 8). Laminin was absent from profiles of long-spacing collagen associated with

Figure 8 More advanced form of basal linear deposit labelled with laminin antibodies. Immunogold particles are over flamentous/granular material, but are absent fron amorphous basement membrane-like material. Note absence of labelling from RPE basement membrane (bm) and longspacing collagen (arrowed). The cell basement membrane is shown by arrowheads. $I C L=$ inner collagenous layer, $R P E=$ retinal pigment epithelium (bar $=1 \mu \mathrm{m}$ ).

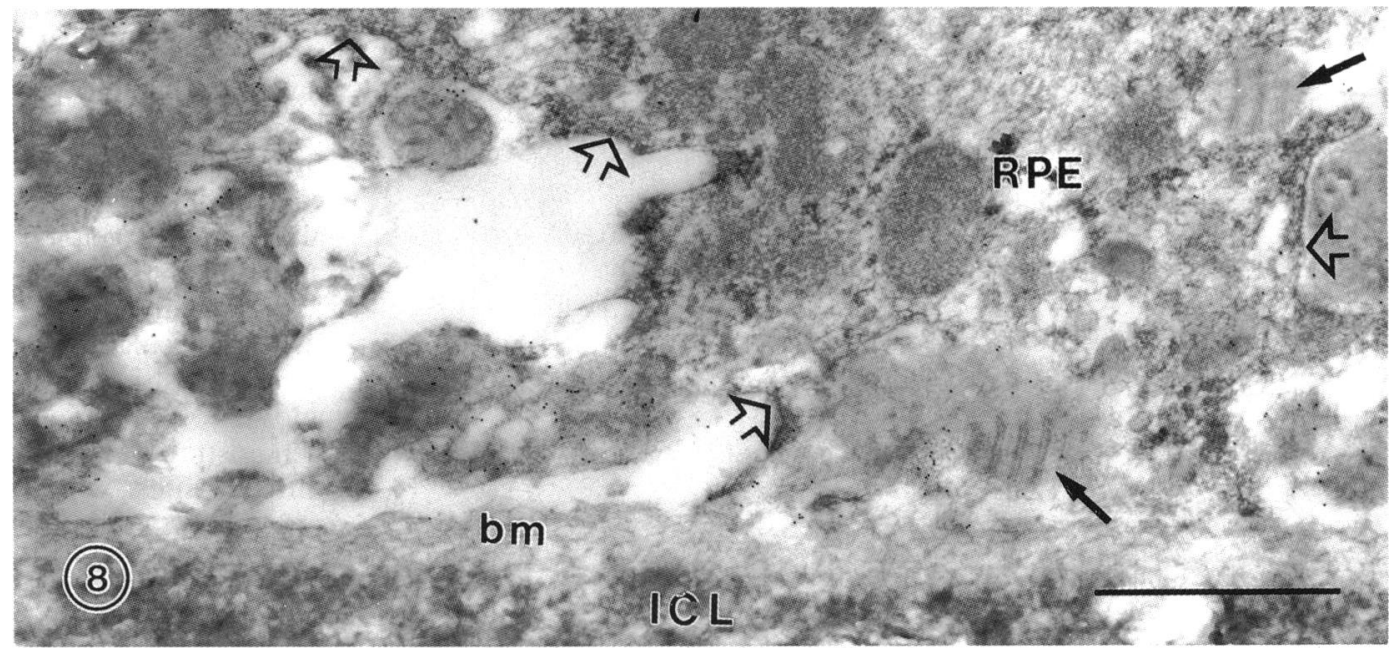


Figure 9 Normal rabbit serum control. Note lack of immunogold particles from basal linear deposit $(B L D)$ and cell nucleus $(\mathrm{Nu})$. Some non-specific labelling is present in Bruch's membrane (Br) $(b a r=1 \mu m)$

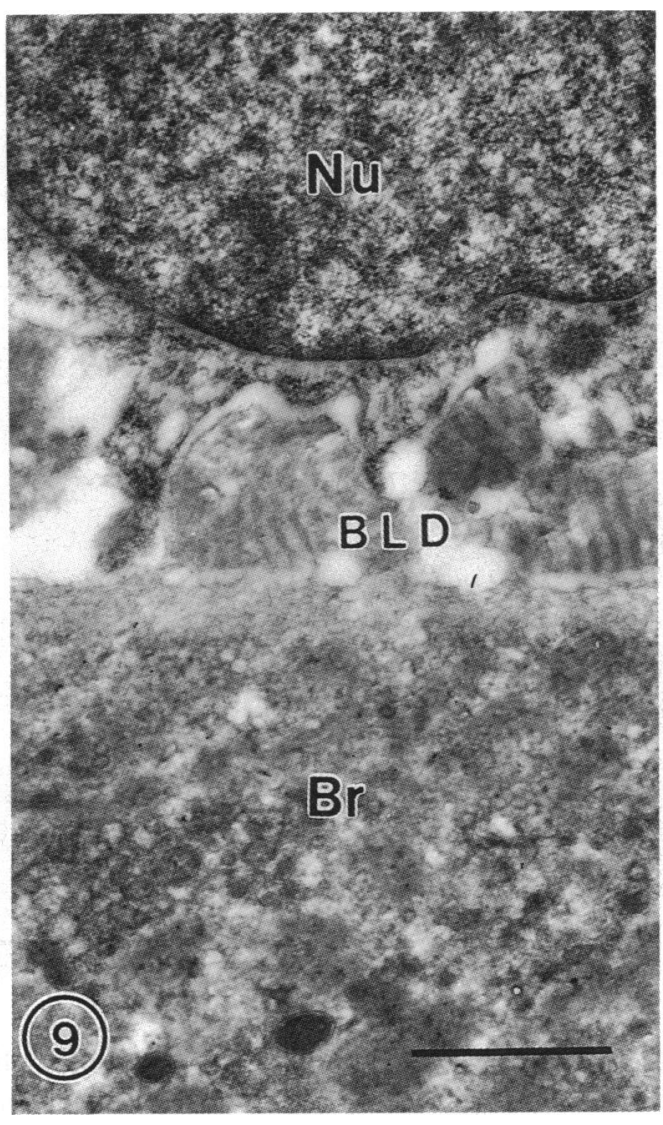

these large basal linear deposits (Fig 8). Immunogold labelling for type IV collagen was not associated with any of the basal linear deposits described above.

CONTROLS

Immunogold labelling was sparse and non-

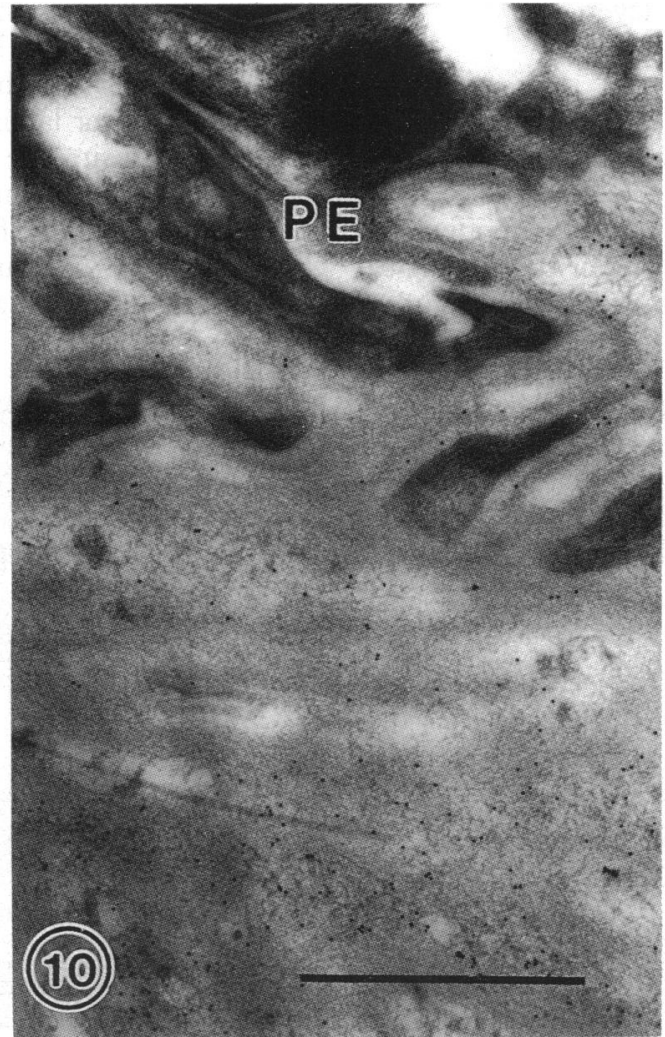

specific in both the normal goat serum and normal rabbit serum negative controls (Fig 9). Non-specific labelling tended to be greater in normal rabbit serum controls than in normal goat serum controls. Type IV collagen was demonstrated in the basement membranes of the retinal vessels (internal positive control). In the ciliary body control tissue, collagen type IV and laminin were observed on the basement membranes of the pigmented and non-pigmented ciliary epithelium (Fig 10). Laminin was also localised to zonular fibres arising from the ciliary body.

\section{Discussion}

MACULA

Previous studies have been conducted on the distribution of collagen type IV and laminin in Bruch's membrane of the human eye $e^{10-12}$ but have not referred to the macular region. To the best of our knowledge the present study is the first to concentrate on normal aged human macula. The importance of the macula in retinal disease merits separate consideration and this study is intended to provide baseline information for further investigation of age-related macular degeneration. It cannot be assumed that the distribution of extracellular matrix components in the macular choroid and RPE is identical to that of the extramacular regions, particularly since there are obvious differences in functional anatomy.

\section{CHOROID}

The overall distribution of type IV collagen seen in our aged macular tissue was similar to that of Das et $a l^{11}$ in their fine structural immunogold study of extramacular human tissue. In their study and the present study labelling was more intense in the basement membrane of the choriocapillaris when compared with that of the RPE basement membrane. A similarity was also observed in the small amount of labelling of the collagenous layers.

Das et $a l^{11}$ also localised laminin to the choriocapillary and RPE basement membranes but labelling was 'far less intense than that seen with type IV collagen'. In our study laminin labelling of these two basement membranes in the macular region was of insufficient intensity to be considered as positive. Laminin, a large multidomain glycoprotein, is a major structural basement membrane component ${ }^{30}$ and numerous biological functions of basement membranes are controlled by its presence..$^{190}$ However, although collagen type IV, laminin, nidogen, and heparan sulphate proteoglycan are the best known components of basement membranes, the molecular architecture of basement membranes is incompletely understood. ${ }^{30}$ In addition, a variation in composition has been demonstrated in basement membranes from different sites. ${ }^{30}$ The localisation of laminin both to discrete structures within Bruch's membrane and to basal linear deposit is unique to the present study.

Light microscopic immunohistochemistry of 
human choroid indicated the presence of both type IV collagen and laminin in Bruch's membrane. ${ }^{10}{ }^{12}$ Newsome et $a l,{ }^{10}$ in an immunofluorescene study, stated that there was a bilaminar staining pattern for these two components. Unfortunately, the lack of resolution with immunofluorescent labelling does not permit a distinction between the constituent layers of Bruch's membrane. The bilaminar immunofluorescent pattern can be explained by an early deposition of basal linear deposit on the basal aspect of the RPE cells and in granular deposits in close apposition with the choriocapillaris as demonstrated by electron microscopy in the present study. There are a large number of alternative explanations for the differences in results obtained by immunocytochemistry, such as loss of antigenicity due to fixation, relocation of antigen in unfixed material, and differences in antibody specificity.

\section{BASAL LINEAR DEPOSIT}

Various types of drusen and basal linear deposit have been recognised histopathologically but clinicopathological correlations have been sparse. ${ }^{1}$ At present no satisfactory classification exists and identification of the constituents of these deposits may elucidate their origin. Disciform macular degeneration represents a response of the posterior pole tissues to pre-existing disorder of the subretinal structures. For example, one proposal is that the process leading to disciform degeneration begins with thickening of the inner aspect of Bruch's membrane owing to synthesis of abnormal basement membranelike material. ${ }^{1}$

Though basal linear deposit, ${ }^{3}$ or alternatively, diffuse soft drusen, ${ }^{31}$ has been recognised by light microscopists for many years and the fine structure was first described in 1964 by Lerche, the nature remains enigmatic. Sarks ${ }^{3-4}$ suggested that basal linear deposit represents a progressive disturbance in the metabolism of the RPE and that it may exert an influence on vascular ingrowth in disciform degeneration. ${ }^{3}$ In its earliest form basal linear deposit is similar in morphology to basement membrane material and it is acceptable to speculate that basal linear deposit is a secretory product of RPE cells. ${ }^{2}$ In more advanced stages basal linear deposit appears to be augmented by cellular deposit derived mainly from RPE cell membranes ${ }^{2}$ and longspacing collagen. ${ }^{33}$ The association of laminin with two constituents of basal linear deposit was clearly demonstrated in the present study. Though in this investigation early forms of basal linear deposit possessed a similar morphology to that of basement membranes, type IV collagen was absent from these deposits. Thus basal linear deposit was biochemically distinct from the RPE basement membrane since the latter structure possessed a variable amount of type IV collagen but not laminin.

Laminin was a component of all forms of basal linear deposit which was observed between the RPE cell membrane and its basement membrane. Since laminin is an extracellular product as distinct from a cellular constituent, it can be concluded that early forms of basal linear deposit are quite distinct from a mere deposition of cell breakdown products. It is also reasonable to assume that laminin is a disordered synthetic product of RPE cells due to its close proximity to the basal cytoplasmic membrane.

\section{LAMININ IN BRUCH'S MEMBRANE}

The occurrence of laminin in deposits in the inner collagenous layer and between the outer collagenous layer and the basement membrane of the choriocapillaris appears to be unique to the aged macula. As these deposits were considerably more common in intercapillary spaces, they may represent remnants of retreating choriocapillaris. Alternatively the deposits may be the product of a senescent RPE. The latter theory is supported by embryonic studies which have shown that in the development of Bruch's membrane there is an extension outward one layer at a time from the basement membrane of the RPE. ${ }^{34}$ Since the RPE has been shown in cell culture studies to produce all of the extracellular matrix components present in Bruch's membrane it is reasonable to assume that much, if not all, of Bruch's membrane is laid down by the RPE. ${ }^{12}$ It is therefore conceivable that laminin deposits observed in our study within Bruch's membrane are the synthetic products of RPE cells and are not derived by diffusion from the choriocapillaris. It may be that such deposits are peculiar to aging and in being secreted in a monomeric form have polymerized within Bruch's membrane. On no occasion was an asssociation observed between laminin and the elastic tissue either in Bruch's membrane or in the supply vessels of the macular choroid.

In previous studies of aged human ciliary body and outflow system we noted plaques of extracellular matrix material in the tips of longitudinal ciliary muscle fibres ${ }^{29}$ and in the cribriform layer. ${ }^{26}$ These plaques bear a striking resemblance to the early forms of basal linear deposit seen in this study and in each location the extracellular matrix deposits were morphologically similar. The immunolabelling patterns for laminin and type IV collagen were also similar. It is interesting to speculate that since in all three regions the tissues act as a barrier to fluid drainage, metabolic byproducts may stimulate extracellular matrix deposition.

Finally, it is appropriate to consider the significance of the present study in relation to adhesion of the RPE to Bruch's membrane. The occurrence of discrete patches of laminin labelling between the inner collagenous layer and the RPE basement membrane could be attributed to the presence of anchoring plaques in a rudimentary basement membrane complex. We have observed a similar labelling pattern in the corneal epithelial basement membrane complex. ${ }^{27}$ That the RPE is firmly adherent to its basement membrane is highlighted in the clinical condition of RPE detachment: here the site of cleavage occurs between the RPE basement membrane and the inner collagenous layer rather than between the RPE and its basement membrane. ${ }^{35}$ This preferential adherence has been confirmed by scanning electron microscope studies of RPE exposed to trypsin digestion: the 
basement membrane separated from the inner collagenous layer rather than the RPE. ${ }^{35}$ Firm adherence of the RPE to its basement membrane is presumably mediated by hemidesmosomes which have been described in the basal cell processes of the RPE. ${ }^{36}$ This in turn would suggest the existence of a basement membrane complex with anchoring fibrils and anchoring plaques such as that seen in corneal epithelium. ${ }^{37}{ }^{38}$ In an immunoelectron microscope study of aged human cornea we were able to demonstrate the presence of laminin in the anchoring plaques of the basement membrane complex. ${ }^{27}$ We would therefore suggest that labelling observed between the RPE basement complex and the inner collagenous zone may be due to the presence of anchoring plaques.

We are pleased to acknowledge the technical assistance of Mrs Sophia Cameron. The efficiency of $\mathrm{Dr} T$ Kardasopoulos in providing the autopsy specimens is greatly appreciated. This work providing the autopsy specimens is greatly appreciated. This work
was supported by the RNIB and the Sir Jules Thorn Charitable was suppo
Trust.

1 Green WR, McDonnell PJ, Yeo JH. Pathologic features of senile macular degeneration. Ophthalmology 1985; 92: 61527.

2 Löffler KU, Lee WR. Basal linear deposit in the human macula. Graefes Arch Clin Exp Ophthalmol 1986; 224: 493501.

3 Sarks SH. New vessel formation beneath the retinal pigment epithelium in senile eyes. Br F Ophthalmol 1973; 57:951-65.

4 Sarks SH. Ageing and degeneration in the macular region: a clinico-pathological study. Br f Ophthalmol 1976; 60: 324 41 .

5 Killingsworth MC. Age-related components of Bruch's membrane in the human eye. Graefes Arch Clin Exp Ophthalmol 1987; 225: 406-12.

6 Newsome DA, Huh W, Green WR. Bruch's membrane agerelated changes vary by region. Curr Eye Res 1987; 6: 1211related.

7 Hogan M. Bruch's membrane and disease of the macula. Role of elastic tissue and collagen. Trans Ophthalmol Soc UK 1967; 87: 113-67.

8 Feeney-Burns L, Ellersieck M. Age-related changes in the ultrastructure of Bruch's membrane. Am $\mathcal{F}$ Ophthalmol 1985; 100: 686-97.

9 Pauleikhoff D, Harper CA, Marshall J, Bird AC. Aging changes in Bruch's membrane: a histochemical and morphologic study. Ophthalmology 1990; 97: 171-8.

10 Newsome DA, Hewitt AT, Huh W, Robey PG, Hassell JR. Detection of specific extracellular matrix molecules in drusen, Bruch's membrane and ciliary body. Am $\mathcal{F}$ Ophthalmol 1987; 104: 373-81.

11 Das A, Frank RN, Zhang NL, Turczyn TJ. Ultrastructural localization of extracellular matrix components in human retinal vessels and Bruch's membrane. Arch Ophthalmol 1990; 108: 421-9.

12 Campochiaro PA, Jerdan JA, Glaser BM. The extracellular matrix of the human retinal pigment epithelium cells in vivo and its synthesis in vitro. Invest Ophthalmol Vis Sci 1986; 27: and its synth

13 Lin WL. Immunogold localisation of extracellular matrix molecules in Bruch's membrane of the rat. Curr Eye Res 1989; 8: 1171-8.

14 Pino RM, Essner E, Pino LC. Location and chemical composition of anionic sites in Bruch's membrane of the rat. f Histochem Cytochem 1982; 30: 245-52.
15 Grindle CFJ, Marshall J. Ageing changes in Bruch's membrane and their functional implications. Trans Ophthalmol Soc UK 1978; 98: 172-5.

16 Gass JDM. Drusen and disciform macular detachment and degeneration. Arch Ophthalmol 1973; 90: 206-17.

17 Leibowitz HM, Kruger DE, Maunder LR, et al. The Framingham eye study monograph: VI Macular degeneration. Surv Ophthalmol 1980;24 (suppl): 428-57. 18 Bird AC. Recent advances in the treatment of senile disciform
macular degeneration by photocoagulation. Brf Ophthalmol 1974; 58: 367-76.

19 Herbst TJ, McCarthy JB, Tsilibary EC, Furcht LT. Differential effects of laminin, intact type IV collagen, and specific domains of type IV collagen on endothelial cell adhesion and migration. $\mathcal{F}$ Cell Biol 1988; 106: 1365-73.

20 Panayotou G, End P, Aumailley M, Timpl R, Engel J. Domains of laminin with growth-factor activity. Cell 1989; 56: 93-101.

21 Kleinman HK, Sephel GC, Tashiro KI, Weeks BS, Burrows BA, Adler SH, et al. Laminin in neuronal development. Ann BA, Adler SH, et al. Laminin in

22 Zabrenetzky VS, Kohn EC, Roberts DD. Suramin inhibits laminin- and thrombospondin-mediated melanoma cell laminin- and thrombospondin-mediated melanoma cell
adhesion and migration and binding of these adhesive adhesion and migration and binding of these adh

proteins to sulfatide. Cancer Res 1990; 50: 5937-42.
23 Marshall GE, Konstas AGP, Lee WR. Immunogold ultrastructural localization of collagens in the aged human outflow system. Ophthalmology 1991; 98: 692-700.

24 Newman GR, Jasani B, Williams ED. A simple postembedding system for the rapid demonstration of tissue antigens under the electron microscope. Histochem $\mathcal{F}$ 1983; 15: 543-55.

25 Marshall GE, Konstas AG, Lee WR. Ultrastructural distribution of collagen types I-VI in aging human retinal vessels. Br F Ophthalmol 1990; 74: 228-32.

26 Marshall GE, Konstas AG, Lee WR. Immunogold localization of type IV collagen and laminin in the aging human outflow system. Exp Eye Res 1990; 51: 691-9.

27 Marshall GE, Konstas AG, Lee WR. Immunogold fine structural localization of extracellular matrix components in aged human cornea. Part 1. Types I-IV collagen and laminin. Graefes Arch Clin Exp Ophthalmol 1991; 229: 15763.

28 Marshall GE, Konstas AGP, Bechrakis NE, Lee WR. An immuno-electron microscope study of the aged human lens capsule. Exp Eye Res 1992; 54: 393-401.

29 Marshall GE, Konstas AGP, Abraham S, Lee WR. Extracellular matrix in aged human ciliary body: an immunoelectron microscope study. Invest Ophthalmol Vis $\mathrm{Sci}$ 1992; 33: 202-16.

30 Yurchenco PD. Assembly of basement membranes. Ann NY Acad Sci 1990; 580: 195-214.

31 Green WR, Key SN. Senile macular degeneration: a histopathological study. Trans Am Ophthalmol Soc 1977; 75: 180254.

32 Lerche $W$. Elektronenmikroskopische Beobachtungen über altersbedingte Veränderungen an der Bruchschen Membran des Menschen. Anat Gesell Verh 1964; 60: 123-32.

33 van der Schaft TL, Mooy CM, de Bruijn WC, Mulder PGH, de Jong PTVM. Histologic features of the early stages of agerelated macular degeneration. Ophthalmology 1992; 99: 27886.

34 Olson MD. Development of Bruch's membrane in the chick: an electron microscopic study. Invest Ophthalmol Vis Sci 1979; 18: 329-38.

35 Goldbaum MH, Madden K. A new perspective on Bruch's membrane and the retinal pigment epithelium. $B r \mathcal{F}$ Ophthalmol 1982; 66: 17-25.

36 Miki H, Bellhorn MB, Henkind P. Specializations of the retinochoroidal juncture. Invest Ophthalmol Vis Sci 1975; 14: 712-7.

37 Keene DR, Sakai LY, Lunstrum GP, Morris NP, Burgeson RE. Type VII collagen forms an extended network of anchoring fibrils. F Cell Biol 1987; 104: 611-21.

38 Brewitt $\mathrm{H}$, Reale E. The basement membrane complex of the human corneal epithelium. Graefes Arch Clin Exp Ophthalmol 1981; 215: 223-31. 